\title{
The SAGA so far: reading the history of the Galaxy with asteroseismology
}

\author{
Luca Casagrande $^{1, a, b}$, Victor Silva Aguirre ${ }^{2}$, Aldo M. Serenelli ${ }^{3}$, Dennis Stello ${ }^{2,4}$, Sofia Feltzing ${ }^{5}$, \\ and Katharine J. Schlesinger ${ }^{1}$ on behalf of the $\mathrm{SAGA}^{5}$ team \\ 1 Research School of Astronomy \& Astrophysics, The Australian National University, ACT, Australia \\ 2 Stellar Astrophysics Centre, Department of Physics and Astronomy, Aarhus University, Denmark \\ 3 Institute of Space Sciences, Campus UAB, Fac. Ciéncies, E-08193 Bellaterra, Spain \\ 4 Sydney Institute for Astronomy, School of Physics, University of Sydney, NSW 2006, Australia \\ 5 Lund Observatory, Department of Astronomy and Theoretical Physics, SE-22100 Lund, Sweden \\ 6 http://www.mso.anu.edu.au/saga
}

\begin{abstract}
Asteroseismology has the capability of delivering stellar properties which would otherwise be inaccessible, such as radii, masses and thus ages of stars. When this information is coupled with classical determinations of stellar parameters, such as metallicities, effective temperatures and angular diameters, powerful new diagnostics for stellar and Galactic studies can be obtained. The ongoing Strömgren survey for Asteroseismology and Galactic Archaeology (SAGA) is pursuing such a goal, by determining photometric stellar parameters for stars with seismic oscillations measured by the Kepler satellite. As the survey continues and expands in sample size, SAGA will provide an unprecedented opportunity to constrain theories of the evolution of the Milky Way disc.
\end{abstract}

\section{Introduction}

The study of the formation and evolution of our Galaxy is entering its golden age, with a number of spectroscopic and photometric surveys targeting one of its main (baryonic) components: stars. Among the latter, red giants are the ideal targets to decipher the formation history of the Milky Way: on the HR diagram they span a vastly different range of gravities and luminosities, thus probing a large range of distances. Their ages essentially cover the entire history of the Universe, thus making them fossil remnants from different epochs of the formation of the Galaxy. The cold surface temperatures encountered in red giants are the realm of interesting atomic and molecular physics shaping their emergent spectra. This temperature regime is also dominated by convection, which is the main driver of the oscillation modes that we are now able to detect in several thousands of stars thanks to space borne asteroseismic missions such as CoRoT and Kepler (e.g., Chaplin \& Miglio, 2013, for a review). By measuring oscillation frequencies in stars, asteroseismology allows us to measure fundamental physical quantities, masses and radii in particular, which otherwise would be inaccessible in single field stars, and which can be used to obtain information on stellar distances and ages (e.g., Silva Aguirre et al., 2011, 2012; Miglio et al., 2013). In particular, global oscillation frequencies not only are the easiest ones to detect and analyze, but are also able to provide the aforementioned parameters for a large number of stars with an accuracy that is generally much better than achievable by isochrone fitting in the traditional sense (see e.g., Silva Aguirre et al., 2013; Lebreton et al., 2014a,b).

Asteroseismology thus provides a powerful and new complementary tool for all past and current photometric and spectroscopic stellar surveys. In fact, while it is relatively straightforward to derive

\footnotetext{
a e-mail: luca.casagrande@anu.edu.au

b $†$ Stromlo Fellow
}

This is an Open Access article distributed under the terms of the Creative Commons Attribution License 4.0, which permits unrestricted use, distribution, and reproduction in any medium, provided the original work is properly cited. 
some sort of information on stellar surface temperature and chemical composition simply from colours and/or spectra (and in many cases even detailed abundances), that is usually not the case when it comes to masses, radii, distances and, in particular, ages. Even when accurate astrometric distances are available to allow comparison of stars with isochrones, the derived ages are still highly uncertain, and statistical techniques are required to avoid biases (e.g., Pont \& Eyer, 2004; Jørgensen \& Lindegren, 2005; Serenelli et al., 2013). Furthermore, isochrone dating is meaningful only for stars in the turnoff and subgiant phase (e.g., Nordström et al., 2004; Casagrande et al., 2011), where stars of different ages are clearly separated in the HR diagram. This is in contrast, for example, to stars on the red giant branch, where isochrones with vastly different ages can fit equally well observational constraints such as effective temperatures, metallicities and surface gravities within their errors (e.g., Soderblom, 2010, for a review).

\section{SAGA}

The purpose of the Strömgren survey for Asteroseismology and Galactic Archaeology (SAGA) is to uniformly and homogeneously observe stars in the Strömgren uvby system across the Kepler field, to derive their classical stellar parameters and thus provide a new benchmark for Galactic studies, similar to the solar neighbourhood. Details on survey rationale, strategy, observations and data reduction are provided in the first SAGA data release (Casagrande et al., 2014a).

Without going into in the gory details of the Strömgren uvby system, here it suffices to say that it was designed for the determination of basic stellar parameters with the ultimate purpose of studying Galactic stellar populations (Strömgren, 1963, 1987), as nicely demonstrated by the GenevaCopenhagen Survey (GCS, Nordström et al., 2004; Casagrande et al., 2011). Indeed, SAGA builds on the legacy of the GCS, representing its natural extension. Similar to the latest revision of the GCS (Casagrande et al., 2011), we combine Strömgren metallicities with broad-band photometry to obtain effective temperatures and metallicities for all targets via the Infrared Flux Method (Casagrande et al., 2006, 2010, 2014b). This facilitates the task of placing SAGA and the GCS on the same scale. However, there are also marked differences between the two surveys: the GCS is an all-sky, shallow survey limited to main-sequence and subgiant stars closer than $\simeq 100 \mathrm{pc}$ (40 pc volume limited). The Kepler targets observed by SAGA are primarily giants located between $\simeq 1$ and $\simeq 6 \mathrm{kpc}$ in a specific region of the Galactic disk, across the Orion arm and edging toward the Perseus arm. The use of giants as probes of Galactic Archaeology is possible since it is relatively straightforward to derive ages for these stars once classical stellar parameters are coupled with asteroseismology. This was not the case for the GCS, where isochrone fitting was used, and thus limited to main-sequence and subgiant stars with known astrometric distances. On the other hand, stars in the GCS have kinematic information, which is not available for the SAGA targets. The different distance ranges sampled by the GCS and SAGA makes them complementary: the stellar properties measured within the solar neighborhood in the former survey can be dynamically stretched across several kpc using kinematics. In contrast, the larger distance range sampled by the giants in SAGA provides in situ measurements of various stellar properties over $\simeq 5 \mathrm{kpc}$.

Observations are being conducted with the Wide Field Camera on the 2.5-m Isaac Newton Telescope (INT), which in virtue of its large field of view and pixel size is ideal for wide field optical imaging surveys. The purpose of SAGA is to obtain good photometry (i.e. few hundredths mag) for all stars in the magnitude range where Kepler is able to measure oscillations, i.e. $10 \lesssim y \lesssim 14$. This requirement can be easily achieved with short exposures on a $2.5-\mathrm{m}$ telescope and indeed all stars for which Kepler measured oscillations are essentially detected in our survey (with a completeness $\gtrsim 95 \%$ ). Strömgren standard stars are chosen from the list of Schuster \& Nissen (1988), which is carefully tied to the system used by Olsen (1983) and underlying the GCS used for our previous investigation of stellar properties in the Galactic disk.

Our images are pre-processed with the Wide Field Survey Pipeline provided by the Cambridge Astronomical Survey Unit (Irwin \& Lewis, 2001). The operations applied to the images consist of debiasing, trimming, flat fielding, and correction for non-linearity. After this pre-processing, we have developed a fast and efficient custom pipeline for the source detection, astrometric solution and photometric calibration of standard and science targets as detailed in Casagrande et al. (2014a). SAGA is 

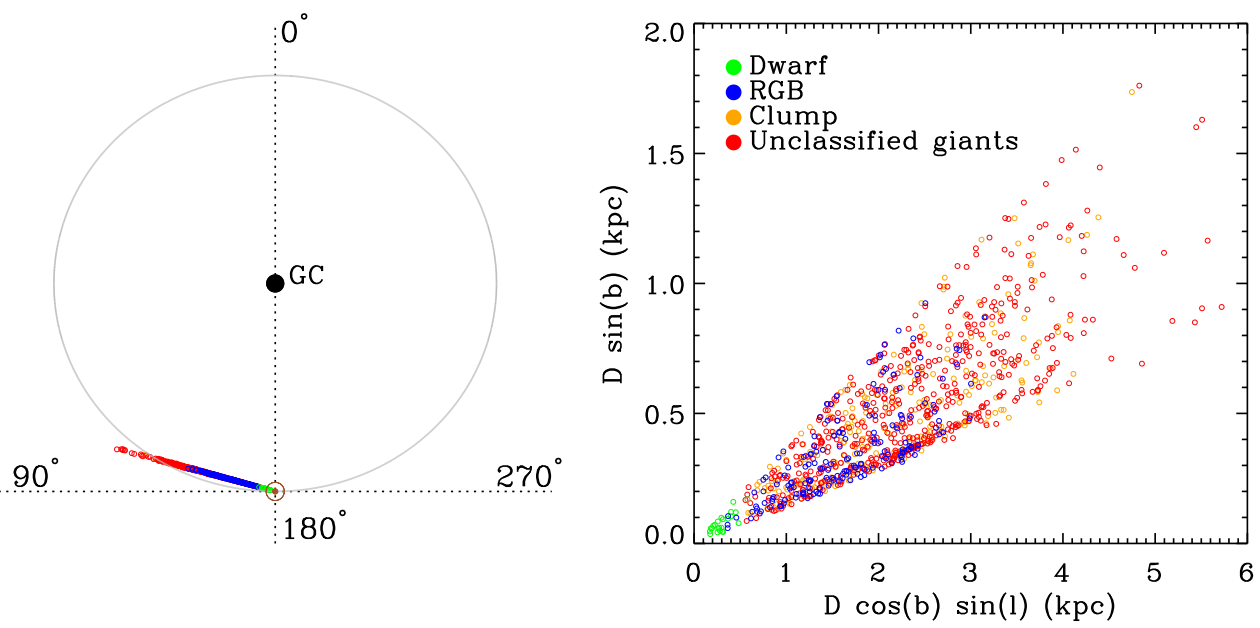

Fig. 1. Location of SAGA targets in the Galaxy. Stars with different seismic classification have different colours, as labelled. Left panel: face-on view in Galactic coordinates, where the distance of each seismic target from the Sun $(D)$ is projected along the line of sight $D \cos (b)$ having direction $l \simeq 74^{\circ}$ and Galactic latitude $b$. The distance between the Galactic Centre (GC) and the location of the Sun $(\odot)$ is marked by the solar circle (in gray). Galactic longitudes $(l)$ at four different angles are indicated. Right panel: same stars as function of Galactic height $\mathrm{Z}=D \sin (b)$ and projected across the $l=90^{\circ}$ direction.

magnitude complete to about $y \simeq 16$ mag, and stars are still detected at fainter magnitudes $(y \simeq 18)$, although with increasingly larger photometric errors and incompleteness. As part of SAGA we are also obtaining photometry on the brightest targets using the four-channel photometer at San Pedro Mártir Observatory.

With SAGA it is thus straightforward to build a magnitude complete and unbiased photometric catalog down to $y \simeq 16$ mag, against which we can benchmark the sample of stars with measured Kepler oscillations. This makes our photometric survey unique in terms of recovering the Kepler selection function of seismic targets (Casagrande et al., 2015). In fact, the selection criteria of the Kepler mission were designed to optimize the scientific yield of the mission with regard to the detection of Earth-size planets in the habitable zone of stars (Batalha et al., 2010). Thus, while the selection function is known for exoplanetary studies, this is not the case when it comes seismic targets, entries in the seismic sample of giants being based on a number of heterogeneous criteria (e.g., Huber et al., 2010). Our approach thus complements other ground based follow up studies of asteroseismic targets (see e.g. Casagrande \& VandenBerg, 2014; Casagrande, 2014c, for the rationale behind photometric parameters and a brief discussion of pros and cons between photometric and spectroscopic surveys). In future observing runs we plan to extend some of our pointings also to $K 2$ fields (Howell et al., 2014).

\section{Stellar parameters and Galactic structure}

The first SAGA data release combines asteroseismic and photometric stellar parameters for 989 Kepler targets, most of which are red giants. The location of the targets in the Galaxy is shown in Figure 1. Within SAGA a novel approach is developed to derive classical and asteroseismic stellar parameters in a fully self-consistent way: for each target, its photometric effective temperature and metallicity, together with the mass, radius, surface gravity, density and distance is computed. For a large fraction of objects, evolutionary phase classification tells whether a stars is a dwarf, is evolving along red giant branch (RGB) or is already in the clump phase (e.g. Stello et al., 2013). The latter distinction is particularly important to derive reliable stellar ages, in particular to select only bona-fide lower RGB stars, where the effect of mass loss is negligible (e.g. Silva Aguirre et al., 2014). 
We provide a careful assessment of random and systematic uncertainties on our parameters. Total uncertainties are of order $82 \mathrm{~K}$ in effective temperature, 0.17 dex in metallicity, 0.006 dex in surface gravity, $1.5 \%$ in stellar density, $2.4 \%$ in radius, $3.3 \%$ in distance and $6.0 \%$ in mass. Age uncertainties vary depending an the availability of seismic classification or not, but are usually below $30 \%$ (Casagrande et al., 2014a, 2015). Confidence in the achieved precision is corroborated by the detection of the first and secondary clumps in a population of field stars and by the negligible scatter in the seismic distances and ages among NGC 6819 member stars (one of the four open clusters located in the Kepler field).

With the stellar parameters derived so far, we are thus in the position of using Kepler targets, and asteroseismology, to investigate some of the most important constraints on Galactic models, such as the age-metallicity relation, and the vertical structure of the Galactic disk via age, mass and metallicity gradients (Casagrande et al., 2015; Schlesinger et al., 2015). Furthermore, calibrating photometric metallicities and age-dating techniques for the entire photometric sample, the continuation of our Strömgren survey promises a leading role for Galactic studies.

\section{References}

Batalha, N. M., Borucki, W. J., Koch, D. G., et al. 2010, ApJ , 713, L109

Casagrande, L., \& VandenBerg, D. A. 2014, MNRAS , 444, 392

Casagrande, L., Portinari, L., \& Flynn, C. 2006, MNRAS , 373, 13

Casagrande, L., Ramírez, I., Meléndez, J., Bessell, M., \& Asplund, M. 2010, A\&A, 512, A54

Casagrande, L., Schönrich, R., Asplund, M., et al. 2011, A\&A, 530, A138

Casagrande, L., Silva Aguirre, V., Stello, D., et al. 2014a, ApJ , 787, 110

Casagrande, L., Portinari, L., Glass, I. S., et al. 2014b, MNRAS , 439, 2060

Casagrande, L. 2014c, Proc. of the workshop "Asteroseismology of stellar populations in the Milky

Way", (eds. A. Miglio et al.) Astrophysics and Space Science Proceedings, arXiv:1409.2272

Casagrande, L., Silva Aguirre, V., et al, ApJ, submitted

Chaplin, W. J., \& Miglio, A. 2013, ARA\&A, 51, 353

Howell, S. B., Sobeck, C., Haas, M., et al. 2014, PASP, 126, 398

Huber, D., Bedding, T. R., Stello, D., et al. 2010, ApJ , 723, 1607

Irwin, M., \& Lewis, J. 2001, NewAR, 45, 105

Jørgensen, B. R., \& Lindegren, L. 2005, A\&A , 436, 127

Lebreton, Y., Goupil, M.-J., \& Montalban, J. 2014, arXiv:1410.5336

Lebreton, Y., Goupil, M.-J., \& Montalban, J. 2014, arXiv:1410.5337

Miglio, A., Chiappini, C., Morel, T., et al. 2013, MNRAS , 429, 423

Nordström, B., Mayor, M., Andersen, J., et al. 2004, A\&A, 418, 989

Olsen, E. H. 1983, A\&AS, 54, 55

Pont, F., \& Eyer, L. 2004, MNRAS , 351, 487

Schlesinger K. J., Casagrande, L., et al, ApJ, submitted

Schuster, W. J., \& Nissen, P. E. 1988, A\&AS , 73, 225

Serenelli, A. M., Bergemann, M., Ruchti, G., \& Casagrande, L. 2013, MNRAS , 429, 3645

Silva Aguirre, V., Chaplin, W. J., Ballot, J., et al. 2011, ApJ , 740, LL2

Silva Aguirre, V., Casagrande, L., Basu, S., et al. 2012, ApJ , 757, 99

Silva Aguirre, V., Basu, S., Brandão, I. M., et al. 2013, ApJ, 769, 141

Silva Aguirre, V., Ruchti, G. R., Hekker, S., et al. 2014, ApJ , 784, LL16

Soderblom, D. R. 2010, ARA\&A, 48, 581

Stello, D., Huber, D., Bedding, T. R., et al. 2013, ApJ , 765, LL41

Strömgren, B. 1963, QJRAS, 4, 8

Strömgren, B. 1987, NATO Advanced Science Institutes (ASI) Series C, 207, 229 